\title{
Eigenview on Jones matrix models of homogeneous anisotropic media
}

\author{
S. Savenkov \\ Kiev Taras Shevchenko University Radiophysics Department, Vladimirskay, 64, 01033 Kiev, Ukraine
}

\begin{abstract}
The polarization of light when it passes through optical medium can change as a result of change in the amplitude (dichroism) or phase shift (birefringence) of the electric vector. The anisotropic properties of media can be determined from these two optical effects. Our main concern here is to revisit the factor of eigenpolarizations and eigenvalues in modeling of polarization properties of homogeneous media and elucidate certain new features in polarization behavior of birefringent and dichroic media.
\end{abstract}

In general a polarization element has two different eigenpolarizations. Those inhomogeneous polarization elements having only one eigenpolarization are said to have degenerate anisotropy, and we call them degenerate polarization elements.

The Jones matrix providing a full information about what can happen with polarization of light passing through the medium, does not, however, provide clear and immediate insight into the polarization properties. This circumstance outlines the main problem of polarimetry - that is the physical interpretation of the information provided by the elements of the Jones matrix. The solution of this problem requires appropriate matrix models, i.e, Jones matrices, that under some assumptions answer the question how the medium is arranged from polarimetric point of view.

So, our aim here is revisiting of known matrix models of homogeneous anisotropic media to find out what exact conclusions we can deduce basing on each of them about inner arrangement of various classes the homogeneous media from polarization point of view, to outline main features - capabilities and restrictions - of physical interpretation for various models and, finally, to answer the question what combinations of anisotropy make the medium to exhibit dichroic, birefringent, degenerate and some other types of polarization behavior.

Apparently the most perspective for this purpose is an approach basing on solution of spectral problem, i.e. on determination of eigenvalues and eigenpolarizations for given Jones matrix. Eventually, all elementary types of anisotropy being the building blocks of any models are specified by their eigenpolarizations, which can be linear, circular and orthogonal, and eigenvalues, which in its turn can be phase or amplitude multipliers. In addition, those media having only one eigenpolarization are said to have degenerate anisotropy.
The history of matrix models of anisotropic media goes back to the early works of Jones, who in 1941 introduced the formalism of $2 \times 2$ matrix [1] describing the response of anisotropic media to the incident polarized light. Relying on this approach, Hurwitz and Jones proved three so-called equivalence theorems [2] providing the basis of the matrix models for a number of classes of homogeneous anisotropic media.

An important topic repeatedly addressed over the past thirty years has been the physical interpretation of experimentally determined Jones based on the so-called polar decomposition [3]. Through polar decomposition, further insights into the Jones matrices are obtained.

The polar decomposition theorem was introduced to polarization calculus by Whitney in 1971 [4]. However, the usefulness of these decompositions was not further exploited until known paper by Jil and Bernabeu published in 1987 [5].

In accordance with polar decomposition theorem the Jones matrix $\mathbf{T}$ is represented as a product of two factors, called polar factors: a general (elliptical) diattenuator $\mathbf{T}_{\mathbf{D}}$ and a general (elliptical) retarder $\mathbf{T}_{\mathbf{R}}$.

$\mathbf{T}=\mathbf{T}_{D} \mathbf{T}_{R} \quad$ or $\quad \mathbf{T}=\mathbf{T}_{R} \mathbf{T}_{D}^{\prime}$

The general diattenuator $\mathbf{T}_{\mathbf{D}}$ changes only the amplitudes of the components of the electric field vector $[5,6]$; its two electric field eigenpolarizations describe two orthogonal elliptical polarization states that exhibit different transmittances (reflectances) but identical phase shifts upon transmission (reflection). The general retarder $\mathbf{T}_{\mathbf{R}}$ changes only the phases of the components of the electric field vector $[5,6]$; its two electric field eigenpolarizations describe two orthogonal elliptical polarization states that exhibit different phase shifts but identical transmittances (reflectances) upon transmission (reflection).

This is an Open Access article distributed under the terms of the Creative Commons Attribution-Noncommercial License 3.0, which permits unrestricted use, distribution, and reproduction in any noncommercial medium, provided the original work is properly cited. 
General diattenuators and retarders are nondepolarizing optical elements and, by virtue of Eq. (1), can be considered as the two "building blocks" of any nondepolarizing optical system.

Thus, from the polar decomposition we can make the following most general conclusions about inner arrangement of homogeneous anisotropy medium in respect of its effect on incident beam of light. First, Unitary and Hermitian polar factors in general case characterize completely birefringent and dichroic properties of the medium, respectively; second, general (elliptical) birefringence and general (elliptical) dichroism are characterized by orthogonal eigenpolarizations; third, medium may possess nonorthogonal eigenpolarizations in case of it to be characterized by both birefringence and dichroism simultaneous and orientations of birefringence and dichroism do not coincide.

At the same time, the noncommutativity of the matrices in Eq. (1) gives birth, in the general case, to the nonuniqueness of the inverse problem solution. Moreover, the given numerical Jones matrix can itself be insufficient for the unique solution of the inverse problem. A simple but nevertheless descriptive example is the answer to the question whether the observed rotation of a linear polarization (described by the Jones matrix in Eq. (11)) is a result of the optical activity of the medium or of the Faraday effect induced in it. Therefore, the nonuniqueness of the inverse problem in polarimetry is not its deficiency but a natural, intrinsic, property. For the unique solution, one should determine the amount and character of additional information necessary to describe the element completely (uniquely). Moreover, this information can often be of nonpolarimetric (and even nonoptical) nature.

The main problem concerning with interpretation of the polar decomposition, to our opinion, is an interpretation of Hermitian polar factor. The matter is that all existed ways of interpretation of the Hermitian polar factor were built on basis of linear amplitude, linear and circular phase anisotropy and do not conclude the circular amplitude anisotropy. In spite of the fact that effect of the polarization element characterized by circular amplitude anisotropy on polarization of incident light can undoubtedly be formally interpreted on basis of certain arrangements of polarization elements characterized by linear amplitude, linear and circular phase anisotropy, nevertheless, circular amplitude anisotropy results from the non-locality of the medium response on incident electromagnetic radiation and, of course, can never be physically reduced to the other types of elementary anisotropy. This means that circular amplitude anisotropy has to include in interpretation of either the polar decomposition or any other matrix model of homogeneous medium.

In contrast to the unitary polar factor associated with elliptical phase anisotropy, which permit clear interpretation on basis of both linear and circular phase anisotropy - this is determined by first Jones equivalence theorem - the Hermitian polar factor has not such simple and clear interpretation including both linear and circular amplitude anisotropy. This results in particular from the fact that multiplication of the Hermitivities is generally not a Hermitivity. Furthermore, below we show that there are no any combinations of linear and circular amplitude anisotropy which would be Hermitian.

It is important to note that first and second equivalence Jones theorems are not characterized by this feature. Although not general, the models basing on first and second equivalence theorems are physically clear to interpret. Indeed, firstly, in scope of the both theorems no any types of transcendent transformations have been occurred. Secondly, in spite of formal existence of "right" and "left" decompositions, both of them are physically equivalent, i.e., it can be shown that the values of linear and circular phase in first Jones equivalence theorem and the values of linear (or circular) amplitude and circular phase anisotropy in second Jones equivalence theorem are invariant for "right" and "left" decompositions $[7,8]$.

It was shown that an arbitrary Jones matrix can be presented as the following product of matrices of four polarization elements [9]:

$\mathbf{T}^{G e n}=\mathbf{T}^{C P} \mathbf{T}^{L P} \mathbf{T}^{C A} \mathbf{T}^{L A}$

These four elements have been for a long time recognized as basic (or simple) polarization elements: linear polarizer $\mathbf{T}^{L A}$, circular polarizer $\mathbf{T}^{C A}$, retarder $\mathbf{T}^{L P}$, and rotator $\mathbf{T}^{C P}$.

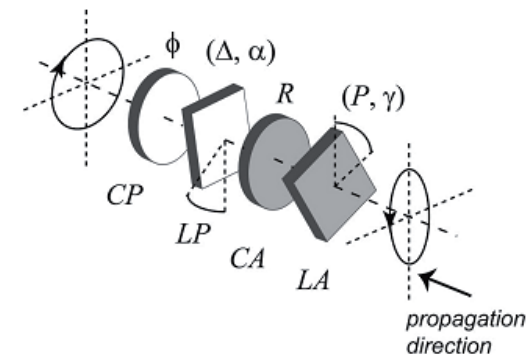

Fig. 1. General polarization element can be presented as a combination of the four polarization elements characterized by elementary types of anisotropy (from right to left): a linear polarizer, a circular polarizer, a retarder, and a rotator.

The corresponding Jones matrices are well-known and can be found elswhere [10].

We wish to note that the matrix model Eq.(2) is general in the sense that the Jones matrix of any nondepolarizing polarization element, regardless of its specific realization or arrangement, can be presented as a combination of the Jones matrices of the four basic elements. This matrix model consists of all four elementary types of anisotropy and is not deduced from the polar decomposition but is a direct generalization of first and second Jones equivalence theorems.

Thus, applying this model we intend to revisit basic types of polarization behavior, namely, birefringence, dichroism and degenerate anisotropy and make an insight to the problem of non-orthogonality of eigenpolarizations. For that end we resort to the equation which defines eigenpolarizations and eigenvalues of homogeneous medium [3]

$$
\mathbf{T E}_{\mathrm{e} 1}=V_{1} \mathbf{E}_{\mathrm{e} 1}, \quad \quad \mathbf{T E}_{\mathrm{e} 2}=V_{2} \mathbf{E}_{\mathrm{e} 2}
$$


The operation of a polarization element through Eq.(3) consists in modification of the polarization state of the incident light by altering the amplitude and phase of its eigenpolarizations separately.

All these scenarios are defined by the eigenvalues. In first case the medium is said to be dichroic and in second - to be birefringent respectively.

Conventional definition of the polarization element to be dichroic is follows [6,11]. It is an element that is characterized only by changes to the amplitudes of the components of the electric vector $\mathbf{E}$, it is also assumed that the isotropic changes to the phase of the components of the electric vector of the incident light are present.

In terms of complex variables [11] it means that the following conditions is satisfied

$$
\chi_{o} / \chi_{o}^{*}=\chi_{i} / \chi_{i}^{*} .
$$

The analysis shows that a dichroic element makes an isotropic phase shift of the incident polarization components only in the basis of its own eigenpolarizations. Therefore, in general the dichroic polarization elements are completely characterized by the following conditions [12]:

$$
\begin{aligned}
& \operatorname{Im}\left(V_{1} / V_{2}\right)=0, \\
& \operatorname{Re}\left(V_{1} / V_{2}\right) \geq 0 .
\end{aligned}
$$

Conditions (5) yield the following form of the corresponding generalized Jones matrix for this class of polarization elements

$$
\mathbf{T}=\frac{\exp (i \phi)}{\chi_{e 1}-\chi_{e 2}}\left(\begin{array}{cc}
|B| \chi_{e 1}-|A| \chi_{e 2} & |A|-|B| \\
-\chi_{e 1} \chi_{e 2}(|A|-|B|) & |A| \chi_{e 1}-|B| \chi_{e 2}
\end{array}\right)
$$

and this Jones matrix is evidently not Hermitian. Thus, arbitrary dichroic medium is generally characterized by non-orthogonal eigenpolarizations. This means that Hermitian polar factor does not characterize completely the dichroic properties of a medium.

Conventional definition of the polarization element to be birefringent is follows [6,11]. It is an element that is characterized only by changes to the phases of the components of the electric vector $\mathbf{E}$, it is also assumed that the isotropic changes of the amplitude of the electric vector of the incident light are present. Thus the birefringent elements can be determined as follows:

$$
I_{o} / I_{i}=\text { const }
$$

where $\mathbf{I}_{\mathrm{i}}$ is the intensity of incident light, $\mathbf{I}_{\mathbf{0}}$ is the intensity of output light, and $\mathbf{V}$ is a constant. A birefringent element is completely characterized by the following conditions [12]:

$$
\begin{aligned}
& \left|V_{1} / V_{2}\right|=1, \\
& \chi_{e 1} \chi_{e 2}^{*}=-1 .
\end{aligned}
$$

This yields the following form of the corresponding generalized Jones matrix for this class of polarization elements (up to isotropic multiplier)

$\mathbf{T}=\left(\begin{array}{cc}\exp (i \phi)+\left|\chi_{e}\right|^{2} & \left|\chi_{e}\right|[\exp (i \phi)-1] \exp (-i \psi) \\ \left|\chi_{e}\right|[\exp (i \phi)-1] \exp (i \psi) & \left|\chi_{e}\right|^{2} \exp (i \phi)+1\end{array}\right)(9)$

Evidently this matrix is Unitary. This result for birefringent elements is consistent with the known results presented elsewhere for the Jones matrix of birefringent medium and, in particular, for the Unitary polar factor of polar decomposition. Essentially, this result exhausts all that can be till now noted about birefringence.

Meanwhile, there exists another scenario. Indeed, as it can be seen from presented consideration the condition of orthogonality of eigenpolarizations is a corollary fact resulting from the requirement of constancy of the ratio of input and output intensities Eq.(7) to be satisfied for all incident polarizations and not by itself a part of definition of birefringence.

Now, consider more weaker requirement on condition (7) - that is let the condition (7) be satisfied not for all input polarizations but at least for eigenpolarizations. Analysis shows that in this case only first relation remains in the conditions (8) and there will be no any conditions on eigenpolarizations.

The Jones matrix of such class of media is follows (up to isotropic multiplier)

$\mathbf{T}=\left(\begin{array}{cc}\left(\chi_{e 1}-\exp (i \phi) \chi_{e 2}\right) & (\exp (i \phi)-1) \\ -(\exp (i \phi)-1) \chi_{e 1} \chi_{e 2} & \left(\exp (i \phi) \chi_{e 1}-\chi_{e 2}\right)\end{array}\right)$.

It can be directly seen that this matrix is not a Unitary (of course, the matrix is not a Hermitian as well). This matrix for all incident polarizations, with the exception of eigenpolarizations, exhibits the dependence of input intensity on incident polarizations. Allowing for exactly this fact, I think that it would be physically adequate to specify this type of polarization behavior not as certain type of birefringence but rather as improper dichroism.

The case when the eigenpolarizations of a given polarization element are coincident, the case of degenerated anisotropy, is follows

$\sqrt{\left(t_{22}-t_{11}\right)^{2}+4 t_{12} t_{21}}=0$

The medium for which the relation (11) is satisfied can not belong either to the class of birefringent or to the class of dichroic media. Relation (11) gives the following evident conditions on eigenpolarizations and eigenvalues of medium to belong to the class of media characterized by degenerate anisotropy:

$V_{1}=V_{2} ; \chi_{1}=\chi_{2}$

The question that arises further is: what combinations of elementary anisotropic mechanisms in a polarization element would make it demonstrate above types of polarization behavior? To answer this question, we resort actually to the matrix model of homogeneous polarization 
element basing on the generalized equivalence theorem presented above, see Eq.(2).

The conditions for a polarization element to have dichroic (13), birefringence (14), and degenerate (15) eigenvalues takes in terms of anisotropy parameters the following forms:

$$
\begin{aligned}
& R(1+P) \cos \frac{\Delta}{2} \sin \varphi+(1-P) \sin \frac{\Delta}{2} \cos (\varphi-2(\alpha-\gamma))=0 \\
& \left((1+P) \cos \frac{\Delta}{2} \cos \varphi-R(1-P) \sin \frac{\Delta}{2} \sin [\varphi-2(\alpha-\gamma)]\right)^{2}- \\
& -4 P\left(1-R^{2}\right)>0 \\
& R(P+1) \cos \frac{\Delta}{2} \sin \varphi-(P-1) \sin \frac{\Delta}{2} \cos (\varphi-2(\alpha-\gamma))=0 \\
& \left((P+1) \cos \frac{\Delta}{2} \cos \varphi+R(P-1) \sin \frac{\Delta}{2} \sin (\varphi-2(\alpha-\gamma))\right)^{2}- \\
& -4 P\left(1-R^{2}\right)<0 \\
& R(P+1) \cos \frac{\Delta}{2} \sin \varphi-(P-1) \sin \frac{\Delta}{2} \cos (\varphi-2(\alpha-\gamma))=0 \\
& \left((P+1) \cos \frac{\Delta}{2} \cos \varphi+R(P-1) \sin \frac{\Delta}{2} \sin (\varphi-2(\alpha-\gamma))\right)^{2}- \\
& -4 P\left(1-R^{2}\right)=0
\end{aligned}
$$

A quite unexpected result is that it turns that the expressions for the eigenvalues to be dichroic, birefringent and degenerate generally comprise all four elementary types of anisotropy and, therewith, all expressions obtained are very symmetrical. They all comprise two conditions, first of them are equal for all types of polarization behaviour and second differs only by sign.

Basing on the matrix model Eq.(2) the generalized conditions for arbitrary polarization element to characterize by orthogonal eigenpolarizations can be derived. These conditions have the form

$$
\begin{aligned}
(1-P)\left[\left(1+R^{2}\right) \cos 2(\alpha-\gamma)-\left(1-R^{2}\right) \cos 2(\alpha-\gamma-\phi)\right]=0 \\
\operatorname{tg}\left(\frac{\Delta}{2}\right)=\frac{1}{2 R} \frac{1-P}{1+P}\left(\left(1+R^{2}\right) \sin 2(\alpha-\gamma)-\right. \\
\left.\quad-\left(1-R^{2}\right) \cos 2(\alpha-\gamma-\phi)\right)
\end{aligned}
$$

From this equations it can be directly seen that the Jones matrix of general, using Shurkliff terminology [10], homogeneous, i.e., those that has orthogonal eigenpolarizations, polarization element firstly - comprise all four elementary types of anisotropy, and secondly - is neither Unitary nor Hermitian.

The fact that there no exist any values of linear $P$ and circular $R$ amplitude anisotropy for combination of these types of anisotropy to have orthogonal eigenpolarizations results directly from above conditions.
As regards the orthogonality of eigenpolarizations in the case of birefringent eigenvalues - this is possible, as expected, only in the case of absence of amplitude anisotropy.

\section{Conclusions}

Above consideration enables to make the following conclusions. The Jones matrices describing all main polarization behaviors, i.e., dichroism, birefringence or degenerate anisotropy, comprise all four elementary types of anisotropy. No any restrictions on eigenpolarizations for polarization elements to be dichroic and degenerate. These classes of polarization elements completely determined by conditions on eigenvalues.

In scope of the case of birefringent eigenvalues there are two possible scenario: first, fulfillment of the requirements of equality of input-output intensities for all input polarizations. In this case we get as a result the orthogonality of eigenpolarizations and Unitarity of the corresponding Jones matrix. Second: fulfillment of the requirements of equality of input-output intensities at least for eigenpolarizations - in this case no any restrictions on eigenpolarizations are posed. Corresponding Jones matrix is neither Unitary nor Hermitian - we specified this case as improper dichroism.

\section{References}

1. R. Jones, J. Opt. Soc. Am. 31, 488 (1941).

2. H. Hurwitz, H., C.R. Jones, J. Opt. Soc. Am. 31, 493 (1941).

3. R. Horn, Ch. Johnson, Matrix Analysis (Cambridge Univ. Press, London, 1986).

4. C. Whitney, J. Opt. Soc. Am. 61, 1207 (1971).

5. J.J. Gil, E. Bernabeu, Optik (Stuttgart) 76, 67 (1987).

6. S.-Y. Lu, R.A. Chipman, J. Opt. Soc. Am. A 13, 1106 (1996).

7. S. N. Savenkov, I. S. Marfin, Proc. of SPIE Vol. 6536, 65360G, (2007)

8. H. Hammer, J. Mod. Opt. 51, 597 (2004).

9. S.N. Savenkov, V.V. Marienko, E.A. Oberemok, O.I. Sydoruk, Phys. Rev. E 74, 056607 (2006).

10. W.A. Shurcliff, Polarized light-production and use (Harvard Univ. Press, Harvard, 1962).

11. R.M. Azzam, N.M. Bashara, Ellipsometry and Polarized Light (North-Holland Publishing Company, New York, 1977).

12. S.N. Savenkov, O.I. Sydoruk, R.S. Muttiah, Appl. Optics, 46, 6700 (2007). 